\title{
Aplicação de CFD para o cálculo de coeficientes de pressão externos nas aberturas de um edifício
}

\author{
Application of CFD simulations for the calculation of \\ external wind pressure coefficients on openings of a \\ building
}

\section{Daniel Cóstola \\ Marcia Peinado Alucci}

\section{Resumo}

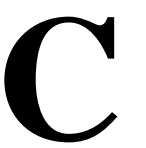

oeficientes de pressão $\left(\mathrm{C}_{\mathrm{p}}\right)$ são um importante parâmetro no estudo da ventilação pela ação dos ventos em edifícios. Este artigo tem por objetivo avaliar a viabilidade no uso de dinâmica dos fluidos computacional (CFD - Computational Fluid Dynamics) para a obtenção de $\mathrm{C}_{\mathrm{p}}$, assim como avaliar a importância de alguns dos aspectos da configuração das simulações nos resultados obtidos. Os seguintes aspectos foram analisados: densidade da malha adotada, sensibilidade ao perfil de vento, e sensibilidade à rugosidade no piso do domínio. São ainda discutidos os valores de $\mathrm{y}^{+}$, assim como a relação entre valores de $\mathrm{C}_{\mathrm{p}}$ médio presentes na literatura $\mathrm{e}$ valores de $C_{p}$ local obtidos nas simulações. Adotou-se nas simulações um edifício isolado de 5 pavimentos, tipicamente utilizado em habitação de interesse social no Brasil. $\mathrm{O}$ artigo conclui que o uso de CFD para definição de $\mathrm{C}_{\mathrm{p}}$ é viável e constitui uma importante alternativa aos túneis de vento. Porém, diferentes configurações da simulação levam a desvios consideráveis, tanto em termos absolutos (até $\pm 0,5$ ) quanto em termos relativos (até 50\%). O teste de independência da malha se mostrou fundamental, assim como a definição do perfil de vento. Futuros trabalhos devem se concentrar na validação das simulações.

Palavras-chave: Coeficiente de pressão. Modelo de rede. CFD. CFX. Habitação de interesse social.

Daniel Cóstola Faculdade de Arquitetura e Urbanismo Universidade de São Paulo Rua do Lago, 876 São Paulo - SP - Brasil CEP 05508-080 Tel.: (11) 3091-4681 r.216 E-mail: daniel_costola@uol.com.br

Marcia Peinado Alucci Faculdade de Arquitetura e Urbanismo Universidade de São Paulo E-mail: marcialu@usp.br

Recebido em 08/03/2010 Aceito em 19/01/2011

\section{Abstract}

Pressure coefficients $\left(C_{p}\right)$ are a key parameter in the study of wind-driven natural ventilation in buildings. This paper reports the results of a research study aimed at evaluating the feasility of using CFD (Computational Fluid Dynamics) to obtain $C_{p}$ data and also evaluating the importance of some of the aspects of the simulation setup in the results obtained. The following aspects are addressed: mesh independence, sensitivity to the wind profile and to the roughness of the domain floor. Values of $y^{+}$are discussed, and surface-averaged $C_{p}$ values from the literature are compared to local $C_{p}$ calculated by $C F D$. The building used in the simulations is a 5 storey, isolated building, typically adopted in social housing projects in Brazil. This paper concludes that CFD simulations are feasible and constitute an important alternative to obtain $C_{p}$ values when compared to wind tunnel experiments. However, differences in the simulation setup may lead to significant deviations in the results, both in absolute values (up to \pm 0.5 ) and in relative values (up to 50\%). The mesh independence test has proven to be fundamental for a reliable simulation. The same applies for the inlet wind profile definition. Future work should focus on the validation of simulations.

Keywords: Pressure coefficient. Airflow network model. CFD. CFX. Social housing. 


\section{Introdução}

Coeficientes de pressão $\left(\mathrm{C}_{\mathrm{p}}\right)$ de um edifício são um dos principais parâmetros no estudo da ventilação natural pela ação dos ventos, sendo um dos dados de entrada de diversos programas de simulação do desempenho térmico do edifício e de cálculo de vazão de ar, tais como EDSL-TAS, Energy Plus, ESP-r, Aiolos e Contam (ORME, 1999; CRAWLEY et al., 2008). $\mathrm{C}_{\mathrm{p}}$ é apontado como um dos parâmetros com maior influência no desempenho térmico do edifício (WIT, 2001). Define-se $\mathrm{C}_{\mathrm{p}}$ como sendo o quociente adimensional entre a pressão dinâmica medida em um ponto $\mathrm{x}$ na fachada do edifício $\left(\mathrm{P}_{\mathrm{x}}\right)$ e a pressão dinâmica do fluxo de ar (vento) não perturbado a barlavento $\left(\mathrm{P}_{\mathrm{d}}\right)$, conforme expressa a Equação 1 . (ETHERIDGE; SANDBERG, 1996; AWBI, 1998a):

$$
C_{p}=\frac{P_{x}}{P_{d}}
$$

Onde:

$$
P_{d}=\frac{\rho \cdot V_{r e f}^{2}}{2}
$$

A pressão dinâmica do fluxo não perturbado $\left(\mathrm{P}_{\mathrm{d}}\right.$ na Equação 2) é a força por unidade de área exercida pelo vento em um plano ortogonal ao sentido do escoamento, na qual: $\mathrm{P}_{\mathrm{d}}$ é a pressão dinâmica do fluxo $(\mathrm{Pa}) ; \mathrm{V}_{\text {ref }}$ é a velocidade do vento tomada na mesma altura do topo do edifício $(\mathrm{m} / \mathrm{s})$; e $\rho$ é a massa específica do ar $\left(\mathrm{kg} / \mathrm{m}^{3}\right)^{1} . \mathrm{C}_{\mathrm{p}}$ varia em função do ponto de interesse na fachada, da forma do edifício, da geometria do entorno e da direção do vento.

Dados de $\mathrm{C}_{\mathrm{p}}$ obtidos em edifícios reais são raramente utilizados em estudos de ventilação e se destinam fundamentalmente à validação de modelos preditivos (RICHARDS; HOXEY; SHORT, 2001; HEIJMANS; WOUTERS, 1995?). $\mathrm{Na}$ prática, valores de $\mathrm{C}_{\mathrm{p}}$ são tradicionalmente obtidos em experimentos em túnel de vento de camada limite (IRMINGER; NØKKENTVED, 1936; JENSEN; FRANCK, 1965; REINHOLD, 1982). Contudo, experimentos em túnel de vento raramente são realizados em projetos de ventilação natural para um edifício específico, em razão dos custos, equipamentos e alto know-how envolvidos (HOLSCHER; NIEMANN, 1998; SIMPSON; WHALLEY, 2009). Uma prática comum na ausência de dados de $\mathrm{C}_{\mathrm{p}}$ para um edifício que se deseja estudar consiste no uso de valores de $C_{p}$ obtidos para outro edifício de formato semelhante.

\footnotetext{
${ }^{1} \grave{A}$ temperatura de $15^{\circ} \mathrm{C}$ e pressão de $1 \mathrm{~atm}$, a massa específica do ar é de $1,2 \mathrm{~kg} / \mathrm{m}^{3}$.
}

Livros-texto e manuais fornecem um número limitado de dados de $\mathrm{C}_{\mathrm{p}}$ (ALLARD, 1998; ASHRAE, 2001; SANTAMOURIS; WOUTERS, 2006; FROTA; SCHIFFER, 2000; BITTENCOURT; CÂNDIDO, 2008). Alguns bancos de dados e modelos preditivos oferecem conjuntos de dados de $\mathrm{C}_{\mathrm{p}}$ médio para as faces de edifícios com formas simples, como cubos, cilindros, paralelepípedos de diferentes proporções e afins (AIVC, 1984; LIDDAMENT, 1986; SWAMI; CHANDRA, 1988; GROSSO, 1992; KNOLL; PHAFF; GIDS, 1995; QUAN et al., 2007). Contudo, é incomum encontrar dados para edifícios de formatos mais complexos ou com entornos de geometria variada, típicos das cidades brasileiras (CÓSTOLA; BLOCKEN; HENSEN, 2009). Além disso, o uso do $C_{p}$ médio da fachada aumenta a probabilidade de erros no cálculo de vazão, apresentando diferenças de até $400 \%$ no fluxo de ar calculado (CÓSTOLA et al., 2010).

Quando a definição de $\mathrm{C}_{\mathrm{p}}$ por meio de túnel de vento não for possível, a dinâmica dos fluidos computacional, mais conhecida pela sigla em inglês CFD (Computational Fluid Dynamics), oferece uma alternativa para a simulação do escoamento ao redor dos edifícios e a obtenção de $\mathrm{C}_{\mathrm{p}}$ (CÓSTOLA; BLOCKEN; HENSEN, 2009). CFD tem sido utilizado para estudar diversos fenômenos relacionados ao escoamento do vento ao redor do edifício (STATHOPOULOS, 1997), tais como transferência de calor (DEFRAEYE; BLOCKEN; CARMELIET, 2010), incidência de chuva nas fachadas (BLOCKEN; CARMELIET, 2006), conforto de pedestres em áreas urbanas (PRATA, 2005), impacto da verticalização na ventilação urbana (PRATA, 2005; LEITE, 2008), balanço térmico de áreas urbanas e ilhas de calor (BRANDÃO, 2009) e ventilação em edifícios (CÂNDIDO; TORRES; BITTENCOURT, 2005; CARDOSO; PESSOA; BITTENCOURT, 2005; CAVALCANTI; CÂNDIDO; BITENCOURT, 2005; FIGUEIREDO, 2008; HOOFF; BLOCKEN, 2010). CFD é uma poderosa ferramenta, porém esse tipo de simulação apresenta diversos desafios tanto com relação à alta capacidade de processamento necessária quanto em relação à configuração das simulações (dados de entrada, parâmetros e modelos adotados).

Com relação à capacidade computacional, o desafio reside na relação entre a dimensão do problema a ser simulado, a capacidade de processamento acessível aos projetistas de ventilação natural (i.e. computadores pessoais) e o tempo de processamento necessário, que deve, em 
princípio, ser compatível com a dinâmica e com os prazos de projetos de arquitetura e engenharia.

Com relação à configuração das simulações, é possível identificar diferenças significativas entre as práticas recomendadas na literatura (CFX, 2003; COST, 2004; FRANKE et al., 2007) e as práticas adotadas em simulações de CFD realizadas tanto por consultorias quanto por parte do meio acadêmico (descritas em alguns dos artigos citados nos parágrafos anteriores). Muitas das práticas recomendadas na literatura acarretam significativo aumento no tempo necessário para configurar e processar as simulações, e por este motivo são frequentemente ignoradas.

Nesse sentido, o objetivo deste artigo é analisar a viabilidade no uso de CFD para o cálculo de $\mathrm{C}_{\mathrm{p}}$, assim como avaliar a importância de algumas das práticas em CFD recomendadas na literatura.

É importante salientar que o artigo não se propõe a fornecer resultados realísticos de $\mathrm{C}_{\mathrm{p}}$, os quais dependem de validação das simulações por meio da comparação com experimentos em escala reduzida e em edifícios reais.

\section{Método}

Nesta pesquisa o software CFX foi adotado nas simulações, mas outros programas de CFD apresentam basicamente as mesmas funções e é possível estender a eles as colocações feitas nos parágrafos seguintes.

Optou-se por contextualizar cada um dos parâmetros e métodos adotados com uma breve descrição teórica, de forma a facilitar o entendimento das decisões tomadas no decorrer da pesquisa e apresentar as principais referências adotadas.

\section{Discretização das equações}

CFX (2003) e COST (2004) aconselham usar discretizações de primeira ordem apenas para estudos iniciais de um problema, e somente utilizar resultados produzidos com discretizações de segunda ordem. No software CFX esta opção está disponível na utilização de high order em advection schemes, a qual foi adotada neste trabalho.

\section{Discretização do espaço (malha)}

Numa simulação de CFD, o volume de ar ao redor do edifício é dividido em uma malha composta de "pequenos" volumes, para os quais são realizados os cálculos de conservação de massa e momento. De forma geral, quanto mais fina a malha, mais precisos são os resultados e maior o tempo e capacidade de processamento necessários para realizar a simulação. A simulação de CFD deve buscar independência do resultado obtido em relação à densidade da malha adotada. Para assegurar essa independência, deve-se proceder sempre a um teste de refinamento da malha e comparação dos resultados: a malha deve ser tal que seu refinamento não cause alteração significativa do resultado encontrado (COST, 2004). O principal sintoma apresentado por uma malha "grossa", ou seja, não refinada o suficiente para garantir independência, é a falta de convergência na simulação.

Nesta pesquisa adotou-se uma malha não estruturada, composta de tetraedros em grande parte do domínio e de células prismáticas junto das fronteiras sólidas. Foram realizadas simulações para determinar a independência dos resultados em relação à malha, resultando na malha da Figura 1. A malha não estruturada não é a mais adequada devido ao aumento da difusividade, o que aumenta os problemas de convergência da simulação. Porém, a criação desse tipo de malha é muito mais simples que a criação de malhas estruturadas, sua utilização é rotineira em atividades de consultoria, e o uso de malhas refinadas resolve em boa parte os problemas de convergência nas simulações.

Nas fronteiras sólidas rugosas, a primeira célula está localizada acima da altura da rugosidade especificada, devido ao uso de wall functions (CFX, 2003; COST, 2004; HARRIES, 2005).

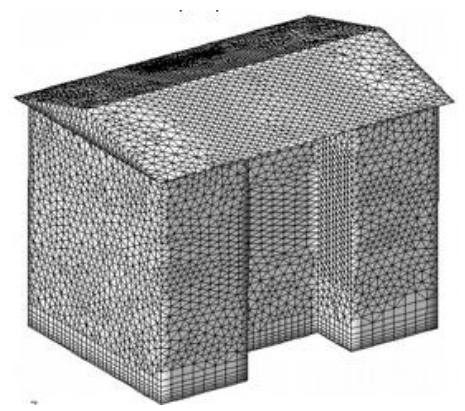

Figura 1 - Malha após refinamento 


\section{Modelagem da turbulência}

A turbulência é um fenômeno físico de alta complexidade que exerce grande influência em escoamentos. Contudo, sua natureza ainda não é totalmente clara e, ao longo dos anos, várias técnicas foram desenvolvidas com o objetivo de calcular seus efeitos no desenvolvimento dos escoamentos (DAVIDSON, 2004). Em geral, programas de CFD fornecem várias alternativas para a modelagem da turbulência, porém a documentação de tais programas fornece pouca ou nenhuma orientação sobre as vantagens e desvantagens de cada alternativa - e.g. CFX (2003).

A abordagem mais comum em simulações de CFD é o uso de equações de conservação simplificadas por meio da técnica conhecida como RANS (Reynolds-averaged Navier-Stokes). RANS permite calcular o escoamento médio sem de fato "resolver" a turbulência, cujos efeitos no escoamento são descritos de maneira simplificada, por meio de um dos chamados modelos de turbulência ou modelos de fechamento. Em termos teóricos, modelos de fechamento de segunda ordem, como o Reynolds Stress Model, são mais abrangentes na descrição das propriedades estatísticas da turbulência, pois permitem levar em conta o aspecto anisotrópico do escoamento. Porém, o desempenho de tais modelos parece não ser sistematicamente superior a modelos de fechamento de primeira ordem em escoamentos ao redor do edifício.

Neste artigo, utiliza-se o modelo de fechamento de primeira ordem standard $\mathrm{k}-\varepsilon$ (CFX, 2003), no qual os efeitos da turbulência no escoamento médio são descritos por meio de duas equações:

(a) uma para a energia cinética turbulenta (k); e

(b) outra à taxa de dissipação da turbulência $(\varepsilon)$.

A opção por esse modelo é baseada em três fatores. Em primeiro lugar, esse modelo de turbulência é largamente utilizado por estudos anteriores sobre escoamentos ao redor de edifícios, tanto em trabalhos acadêmicos, e.g. Yang (2004) e Monteiro et al. (2008), quanto em serviços de consultoria, e.g. Harries (2005). Em segundo lugar, simulações de CFD utilizando modelos de fechamento de primeira ordem (como o standard $\mathrm{k}-\varepsilon$ ) foram validadas com sucesso para diversas aplicações de engenharia do vento (BLOCKEN; CARMELIET, 2006; DEFRAEYE; BLOCKEN; CARMELIET, 2010; HOOFF; BLOCKEN, 2010), ou seja, modelos isotrópicos podem, em certas circunstâncias, fornecer resultados válidos. Um recente projeto internacional sobre boas práticas no uso de CFD para escoamentos ao redor de edifícios
(FRANKE et al., 2007) concluiu que, até o presente momento e no que diz respeito a RANS, não foi possível identificar entre os modelos de turbulência um que seja sistematicamente superior aos demais, seja ele de fechamento de primeira ou de segunda ordem. Por fim, o modelo standard $\mathrm{k}-\varepsilon$ é utilizado devido à relação entre qualidade dos resultados e pequena capacidade de processamento necessária, típica da abordagem RANS. O uso de abordagens alternativas ao RANS, tais como $\mathrm{LES}^{2}$ ou $\mathrm{DES}^{3}$, traz benefícios reconhecidos ao cálculo de $\mathrm{C}_{\mathrm{p}}$, porém ainda é restrito ao meio acadêmico devido às altas capacidades de processamento e armazenamento necessárias (NOZU et al., 2008).

Cabe salientar que, entre os modelos de fechamento de primeira ordem, o standard $\mathrm{k}-\varepsilon$ tem sido identificado como o de pior desempenho, sendo crescente o uso de outros modelos, tais como realizable $\mathrm{k}-\varepsilon$, RNG $\mathrm{k}-\varepsilon$ ou SST k- $\omega$. A modelagem da turbulência é ainda um tema controverso, e o presente artigo não tem por objetivo abordá-lo em profundidade. É altamente recomendável que futuros trabalhos abordem esse tema de maneira sistemática, buscando a melhor relação entre qualidade dos resultados obtidos e capacidade computacional necessária.

\section{Domínio usado nas simulações condições de contorno}

Em uma simulação de CFD, é necessário definir a extensão do volume de ar a ser simulado ao redor do edifício, o domínio. Esse volume deve ser grande o suficiente para que não influencie os resultados obtidos. Nesse caso, foram adotadas as recomendações de COST (2004), que aconselha uma taxa de obstrução inferior a $3 \%$ da seção do domínio, espaçamentos de no mínimo 10 vezes a altura do edifício em todas as direções (exceto na altura), e altura igual a 5 vezes a altura do objeto das simulações (edifício).

A Figura 2 apresenta duas formas de domínio passíveis de utilização em simulações para determinar $\mathrm{C}_{\mathrm{p}}$. $\mathrm{O}$ domínio em forma de paralelepípedo apresenta menor volume e utiliza condições de contorno mais adequadas. Contudo, ele se presta a simular apenas uma direção do vento. O domínio circular é mais utilizado porque permite utilizar a mesma modelagem e malha para simular qualquer direção do vento.

\footnotetext{
${ }^{2}$ LES (Large Eddy Simulation) é uma técnica de CFD na qual os efeitos dos turbilhões de maior energia no escoamento são efetivamente calculados por meio de malhas extremamente densas e simulações em regime transiente.

${ }^{3}$ DES (Detached Eddy Simulation) é uma técnica que combina RANS E LES.
} 

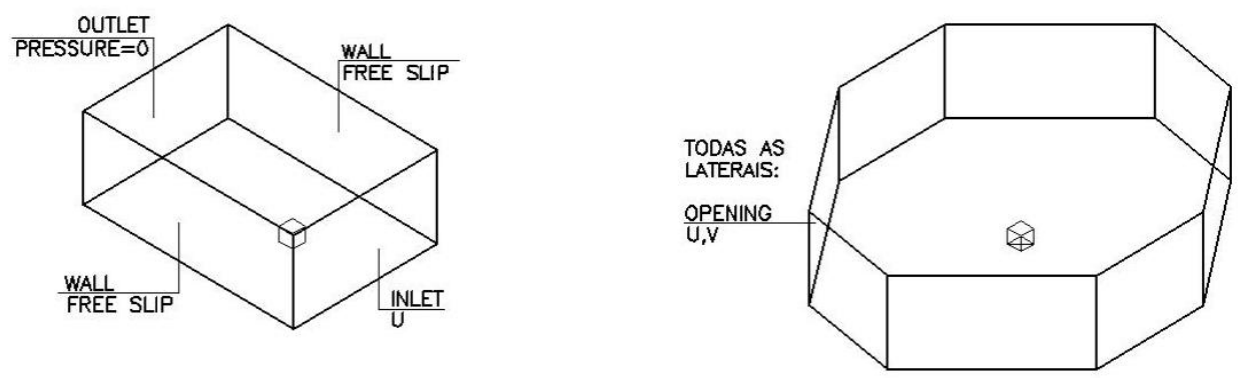

Figura 2 - Condições de contorno para duas formas de domínio possíveis

No caso do domínio circular, as laterais do domínio são configuradas como aberturas, às quais é atribuída uma velocidade nas componentes cartesianas $\mathrm{x}$, y e $\mathrm{z}$. A velocidade na direção $\mathrm{z}$ é igual a zero, e as outras componentes são dadas em variáveis $\mathrm{u}_{1}$ e $\mathrm{v}_{1}$, cujo valor é definido, no caso do CFX, no arquivo externo de configuração (arquivo CCL). Assim, é possível alterar a direção do vento apenas pela edição do arquivo CCL. As desvantagens dessa forma de simulação são o maior número de células e a imposição do perfil de velocidade na saída do domínio, mascarando os efeitos de distâncias inadequadas a sotavento.

Nas simulações utilizou-se um domínio de forma "circular" e adotou-se como condição de contorno nas laterais do domínio um perfil logarítmico correspondendo a CLP neutra (STULL, 1998), sem qualquer correção de caráter topográfico, e com intensidade de turbulência igual a $5 \%$. No CFX, a forma mais adequada de configurar esse perfil é por meio de um arquivo CCL e da inicialização via arquivo BAT (HARRIES, 2005). Um exemplo de arquivo CCL é fornecido em Cóstola (2006).

Com relação às fronteiras sólidas, elas podem ser do tipo free slip, que não oferece resistência ao escoamento, ou no slip, na qual o atrito entre sólido e líquido é considerado e a rugosidade da superfície deve ser especificada.

Na envoltória do edifício e no piso do domínio, vale a condição de não escorregamento. Assim, essas fronteiras foram definidas como no slip. Com relação à rugosidade, as superfícies foram sempre definidas como lisas, com exceção de um grupo de simulações no qual o impacto da adoção de rugosidade no piso foi analisado. No teto do domínio adotou-se a condição de contorno free slip.

Nas fronteiras sólidas do tipo no slip, duas abordagens podem ser adotadas para modelar a interação sólido-fluido: wall functions e low Reynolds number modelling. No caso das wall functions, a interação é calculada em uma única "grande" célula colocada na fronteira sólido- líquido. Nessa célula, a interação é calculada com base em uma função obtida através de um número limitado de experimentos, o que traz vantagens em termos computacionais e permite que alguns aspectos do problema (e.g. rugosidade) sejam descritos por meio de parâmetros. No caso do low Reynolds number modelling, a interação é calculada utilizando-se uma malha muito fina na fronteira sólido-líquido. Por não ser baseada em um número limitado de experimentos, esta abordagem é mais versátil, sendo capaz de captar com maior precisão as interações sólido-líquido. Contudo, esse método exige grande capacidade computacional, e os elementos de rugosidade na superfície devem ser modelados um a um, o que em termos práticos torna esse tipo de simulação viável apenas para superfícies lisas.

Adotou-se nesta pesquisa o uso de wall functions em detrimento do low Reynolds number modelling, pois elas permitem representar os fenômenos na camada limite em função da rugosidade da superfície. $\mathrm{O}$ uso de wall functions permite também o uso de malhas menos densas (menor tempo de processamento) e não representa uma simplificação excessiva para problemas sem transferência de calor e massa (AWBI, 1998b; BLOCKEN; CARMELIET, 2006).

O sucesso no uso de wall functions exige o correto posicionamento dos pontos da malha próximo às fronteiras sólidas. Esse posicionamento deve ser compatível com o fluido e com o gradiente de velocidades presentes na simulação. Para tanto, CFX (2003) recomenda, quando do uso do modelo de turbulência standard $\mathrm{k}-\varepsilon$, que o valor do parâmetro adimensional $\mathrm{y}^{+}$esteja entre 20 e 100 . COST (2004) aconselha um valor entre 30 e 100 para $\mathrm{y}^{+}$. Por sua vez, Blocken, Stathopoulus e Carmeliet (2007) consideram aceitáveis valores entre 30 e 1.000. É possível encontrar diversas referências que indicam diferentes limites para $\mathrm{y}^{+}$, o que demonstra a falta de consenso da comunidade científica em relação aos limites exatos para ele. Contudo, é notável que valores muito baixos $(<20)$ ou muito altos $(>1.000)$ nunca são recomendados. $\mathrm{O}$ valor de $\mathrm{y}^{+}$é um dado de 
saída da simulação, não podendo ser fixado $a$ priori, e será discutido junto com os resultados das simulações.

\section{Convergência}

Simulações de CFD são realizadas por meio da solução iterativa das equações de conservação de massa e momento em cada célula da malha. Essas iterações devem ser repetidas até que se obtenham resultados que respeitem, da melhor forma possível, o princípio de conservação em todas as células. Isso é indicado pelo resíduo do cálculo, i.e. a diferença entre a massa e o momento que entram e que deixam cada célula. $\mathrm{O}$ resíduo de todas as células é combinado em uma única estatística (RMS - Root Mean Square), que indica o nível de convergência da simulação. Nesta pesquisa foi utilizado o critério de convergência recomendado por COST (2004) e CFX (2003). Aconselham que o RMS obtido na simulação seja igual ou inferior a $10^{-4}$. CFX (2003) indica que resíduos acima de $10^{-4}$ são muito pouco confiáveis e servem apenas para fornecer uma ideia superficial sobre o escoamento e para "produzir imagens bonitas".

\section{Operacionalização do cálculo de $C_{p}$}

Uma vez simulado o escoamento ao redor do edifício, o cálculo de $\mathrm{C}_{\mathrm{p}}$ é realizado de maneira similar ao realizado com dados de túnel de vento. No caso do uso do CFX deve-se definir no software de pós-processamento pontos posicionados no centro das aberturas. Os dados de pressão nesses pontos são então exportados para um arquivo de texto. Um ponto adicional deve ser posicionado no fluxo não perturbado antes do edifício, onde são obtidas a velocidade e a massa específica do ar, com os quais a pressão dinâmica é calculada. De posse desses valores calcula-se o $C_{p}$ para os pontos de interesse.

\section{Características das simulações}

Como objeto das simulações, escolheu-se um edifício com 5 pavimentos e planta tipo $\mathrm{H}(18,25$ $\mathrm{m} \times 12,70 \mathrm{~m} \times 15,45 \mathrm{~m}$ ), comumente encontrado nas cidades brasileiras e utilizado em diversos conjuntos habitacionais de interesse social (Figura 3).

As simulações foram processadas em computadores com processador Pentium de $2 \mathrm{GHz}$ e $1 \mathrm{~Gb}$ de memória RAM. Algumas simulações planejadas não puderam ser realizadas devido à falta de capacidade de processamento das máquinas, como as que envolviam grandes extensões de entorno. O processamento em paralelo, que seria a melhor solução para problemas de falta de capacidade de processamento, não foi utilizado devido às dificuldades enfrentadas na configuração do software.

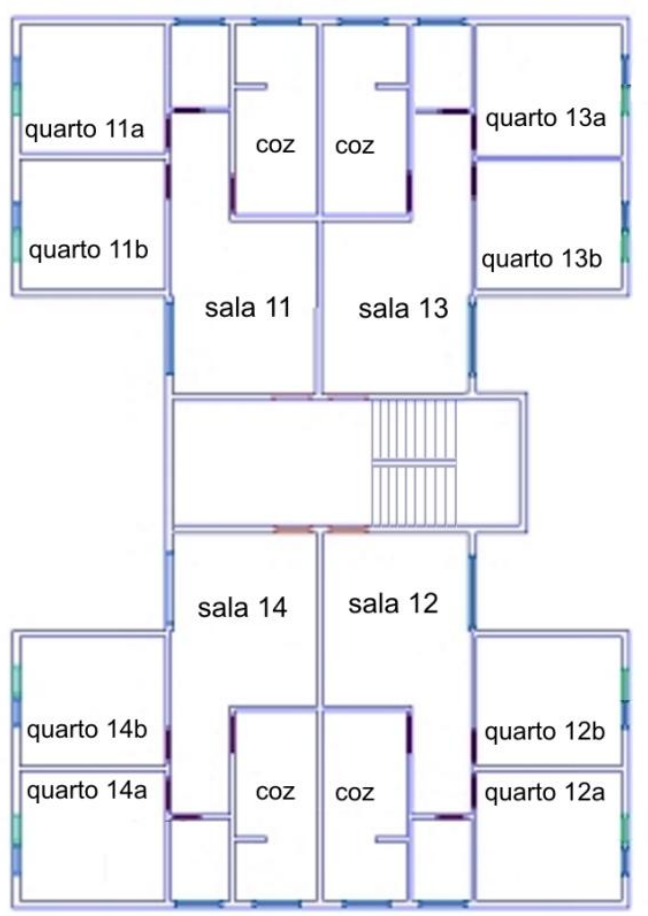

(a)

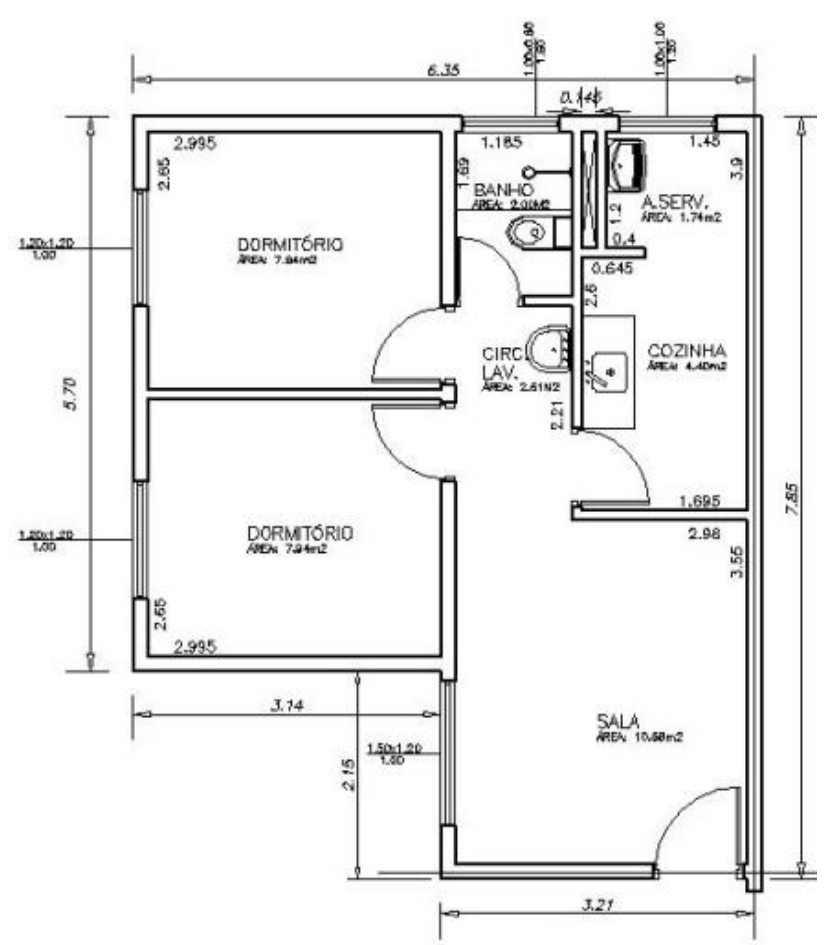

(b)

Figura 3 - Planta tipo H: (a) esquema do andar tipo e (b) planta do apartamento 
Foram realizadas 86 simulações, de forma a explorar um conjunto de parâmetros relevantes em simulações de CFD, que permitiram reproduzir conclusões comumente encontradas na bibliografia.

Em particular, estudou-se o comportamento dos resultados ante as seguintes alterações: direção de incidência do vento, perfil de velocidades médias na entrada e saída do domínio, rugosidade no piso do domínio (entorno do edifício) e resolução da malha utilizada na simulação.

É importante salientar que esses resultados podem apresentar discrepâncias com a realidade, uma vez que eles não foram validados experimentalmente e é conhecida a deficiência na descrição de descolamentos da camada-limite que o modelo de turbulência utilizado (standard $\mathrm{k}-\varepsilon$ ) apresenta.

Nas simulações, os valores de $C_{p}$ foram determinados para ângulos com intervalos de $30^{\circ}$, recomendados em COST (2004), ou seja, para as direções $0^{\circ}, 30^{\circ}, 60^{\circ}, 90^{\circ}, 120^{\circ}, 150^{\circ}, 180^{\circ}, 210^{\circ}$, $240^{\circ}, 270^{\circ}, 300^{\circ}$ e $330^{\circ}$.

\section{Análise dos resultados}

\section{Perfil de velocidades médias do vento}

A Figura 4 apresenta a comparação entre as distribuições de $C_{p}$ na face a barlavento do edifício, obtidas em quatro simulações nas quais foram simulados diferentes perfis de velocidade do vento. Foram utilizados perfis logarítmicos (CLP Neutra) correspondentes a rugosidades variando de 0,001 a 0,3 .

Da análise da Figura 4, que não ilustra a complexidade dos efeitos da mudança de
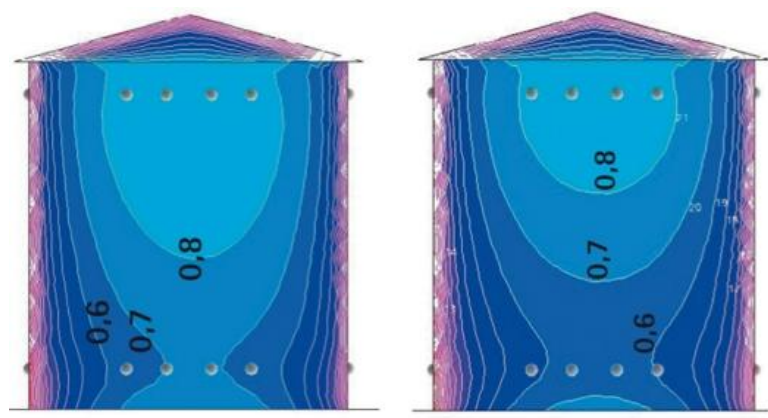

Figura 4 - Distribuição de $C_{p}$ na face a barlavento para perfis de vento com rugosidades de 0,001, 0,01, 0,1 e 0,3
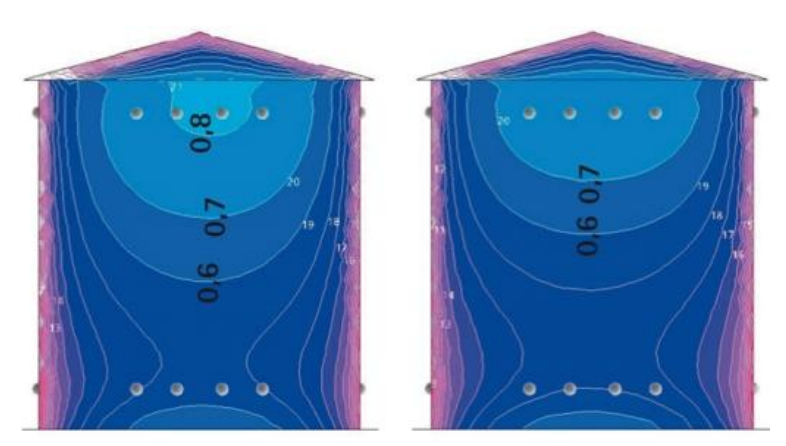

rugosidade nas demais faces do edifício e nas demais direções de incidência do vento, pode-se concluir que o valor de $C_{p}$ é fortemente influenciado pelo perfil de velocidade do vento na entrada e saída do domínio.

Com base nessa constatação, pode-se afirmar que o valor de $\mathrm{C}_{\mathrm{p}}$ para o uso no projeto de ventilação natural deve ser obtido por meio de ensaios ou simulações que considerem a rugosidade do entorno do edifício, e resultados genéricos para perfis de velocidade não especificados podem levar a erros no projeto de ventilação natural.

Ressalta-se que a importância do uso de Cp em estudos de ventilação por ação do vento reside na independência de tal parâmetro em relação à velocidade de referência do vento para edifícios com cantos vivos, que representam a grande maioria das edificações (ETHERIDGE; SANDBERG, 1996). Contudo, essa afirmativa é valida para um edifício com dado entorno, pois variações na velocidade não são acompanhadas de variações no padrão do escoamento, as quais levariam a modificações no $\mathrm{Cp}$. Quando o entorno do edifício é modificado, alterando o perfil de vento, o conjunto do escoamento é também alterado, modificando o valor de $\mathrm{Cp}$, conforme demonstrado na Figura 4.

\section{Rugosidade no piso do domínio}

A Figura 5 apresenta um histograma com as frequências das diferenças entre os valores de $C_{p}$ obtidos para duas simulações que diferem apenas pelo tratamento dado ao piso do domínio: liso, ou com rugosidade igual àquela utilizada para definir o perfil de entrada. No caso, utilizou-se uma rugosidade de $0,1 \mathrm{~m}$. 


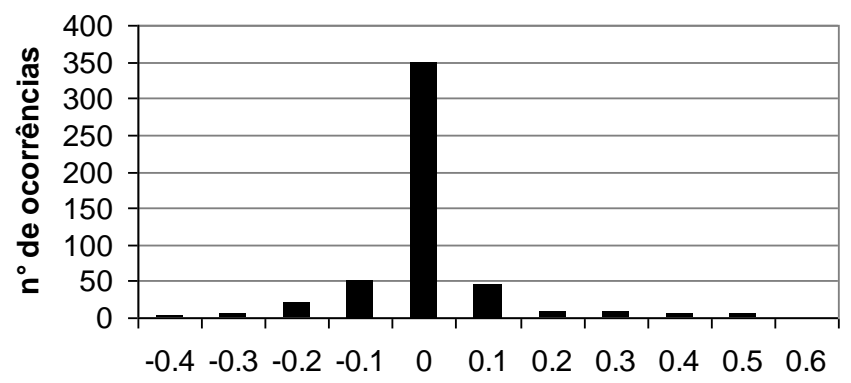

Diferenças entre o $C_{p}$ calculado nas simulações

Figura 5 - Histograma da diferença entre os $C_{p}$ calculados em simulações com e sem rugosidade no piso do domínio
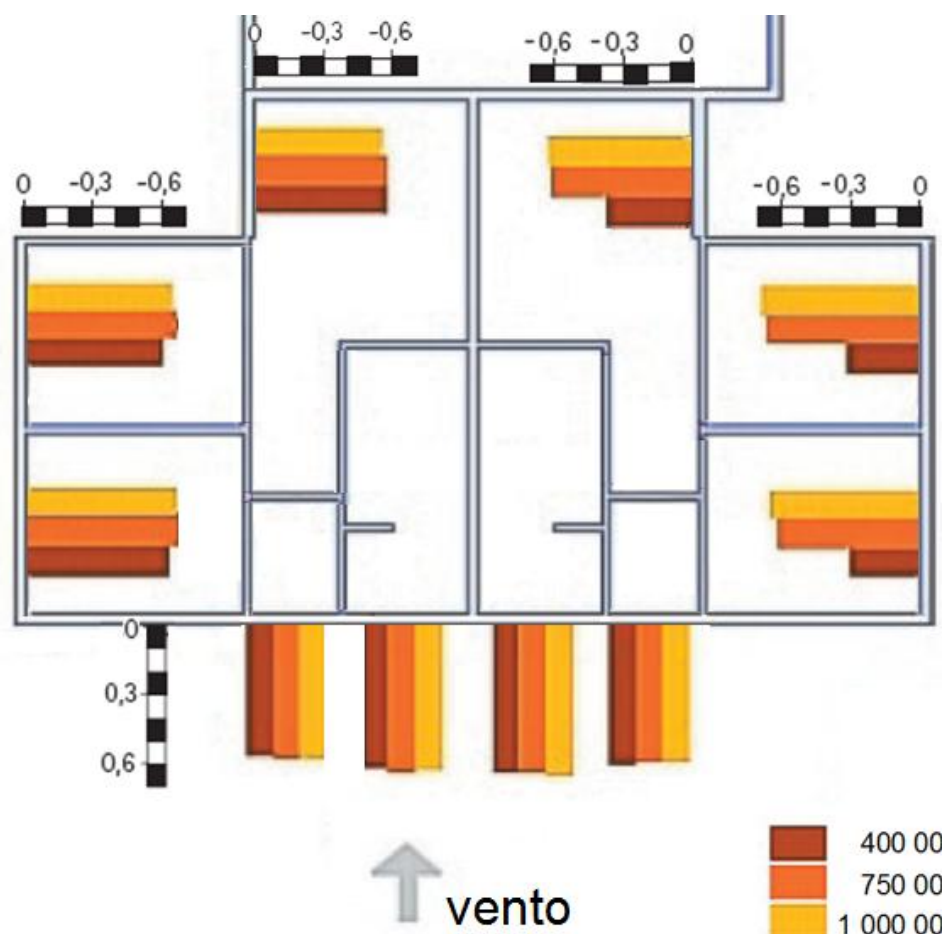

400000 elementos

750000 elementos

1000000 elementos

Figura 6 - Pavimento térreo: comparação entre as simulações com diferentes malhas

Nota-se que na maior parte dos casos (350 de um total de 480 , i.e. $72 \%$ dos casos) não há diferença entre o resultado das simulações com ou sem rugosidade no piso do domínio. Em $10 \%$ dos casos (50), o $\mathrm{C}_{\mathrm{p}}$ é superdimensionado em 0,1 , e em $10 \%$ dos casos, subdimensionado na mesma proporção. Nos $8 \%$ dos casos restantes a diferença entre as simulações ultrapassa 0,1 em módulo, chegando a 0,5 . Pode-se concluir que a presença de rugosidade no piso afeta de maneira significativa o resultado e, portanto, a rugosidade deve ser utilizada. Em áreas com diversos edifícios, a área de piso entre os edifícios deve ser modelada como lisa segundo COST (2004). A despeito da importância desse parâmetro, Blocken, Stathopoulos e Carmeliet (2007) apontam para a incapacidade das wall functions em manter, ao longo do domínio, os perfis de velocidade e turbulência adotados como condição de contorno. Isso indica que ainda não existe consenso quanto à solução a adotar com relação ao tratamento da rugosidade no piso do domínio.

\section{Independência dos resultados em relação à malha}

Na Figura 6, os resultados de três simulações com diferentes malhas são ilustrados por meio de barras, cujo comprimento equivale ao $\mathrm{C}_{\mathrm{p}}$ encontrado para cada uma das janelas do pavimento térreo. As barras escuras representam a simulação com malha mais grossa (400.000 elementos), e as barras claras dizem respeito à simulação com a malha mais refinada (1.000.000 de elementos). Nota-se uma grande diferença (de até $50 \%$ ) entre o comprimento das barras escuras e o das claras, mostrando que esse refinamento provocou grandes alterações nos resultados 
encontrados. A diferença é pequena entre as simulações com 750.000 elementos e 1.000 .000 de elementos, sinalizando a obtenção de independência dos resultados em relação à malha.

A assimetria nos resultados com a malha não refinada (barras escuras) é um claro indicativo da inadequação dessa malha. $\mathrm{Na}$ medida em que a malha é refinada, essa assimetria desaparece. Esses resultados são apenas apresentados neste artigo para salientar a importância da correta configuração da malha. Em termos práticos, apenas os resultados com a malha refinada devem ser reportados e utilizados.

Os resíduos encontrados na simulação com a malha refinada foram próximos a 10-4, indicando que essa malha é a mais adequada. Seria conveniente testar uma ou duas malhas ainda mais refinadas, para que se pudesse estabelecer a malha correta para esta simulação. Contudo, um maior refinamento na malha demandaria capacidade de processamento e memória RAM maiores que as disponíveis. A relação entre memória RAM e tamanho da malha é tal que, de forma geral, $1 \mathrm{~Gb}$ de memória RAM é capaz de simular uma malha com 1 milhão de elementos (HARRIES, 2005). A simulação com malha refinada teve um tempo de processamento total de $2 \mathrm{~h}$ para cada direção do vento.

\section{Simulação da camada limite}

Nas simulações realizadas, o menor valor médio de $\mathrm{y}^{+}$obtido foi de 600 . Esse valor foi encontrado nas simulações com malhas refinadas e com o primeiro ponto da malha a $8 \mathrm{~cm}$ do piso do domínio. Tal valor se deve a dois fatores. O primeiro fator diz respeito ao fato de $\mathrm{o}^{+}$depender da distância adotada na primeira célula, a qual poderia ser reduzida de forma a reduzir $\mathrm{y}^{+}$. Contudo, a primeira célula deve ter altura mínima maior que a rugosidade, o que limita as possibilidades de redução da altura da primeira célula. $O$ segundo fator diz respeito ao fato de $\mathrm{y}^{+}$depender da velocidade de fricção, a qual é baseada na tensão de cisalhamento junto à fronteira sólida, que por sua vez é baseada da velocidade do vento imposta nas laterais do domínio. Considerando-se que $\mathrm{C}_{\mathrm{p}}$ é um valor adimensional tido como independente da velocidade do vento, a redução da velocidade do vento nas laterais do domínio pode ser usada para reduzir o $\mathrm{y}^{+}$. Nas simulações realizadas neste trabalho, utilizou-se uma velocidade de $5 \mathrm{~m} / \mathrm{s}$ a 10 $\mathrm{m}$ de altura em relação ao solo.

$\mathrm{O}$ valor de $\mathrm{y}^{+}$é um indicativo da qualidade da malha próximo a fronteiras sólidas. O teste de independência da malha, ilustrado na Figura 6, indica que a malha adotada é satisfatória e, portanto, indica que a recomendação de y+ em COST (2004) e CFX (2003) é excessivamente rigorosa. Aparentemente, o intervalo sugerido por Blocken, Stathopoulos e Carmeliet (2007) parece mais adequado, uma vez que os resultados obtidos se mostraram independentes da malha. Futuros estudos devem explorar em maior profundidade a importância desse parâmetro.

\section{Importância da posição na fachada nos valores de $C_{p}$}

Conforme descrito na introdução deste artigo, $\mathrm{C}_{\mathrm{p}}$ varia em função do ponto de interesse na fachada (TOLEDO, 1967). Contudo, esse fato é frequentemente ignorado por diversos bancos de dados de $\mathrm{C}_{\mathrm{p}}$, os quais fornecem apenas valores de $\mathrm{C}_{\mathrm{p}}$ médio para a fachada. Cóstola, Blocken e Hensen (2009) descrevem que, por exemplo, os dados de $\mathrm{C}_{\mathrm{p}}$ médio propostos por Liddament (1986) são utilizados em 6 de 10 importantes programas de simulação do desempenho térmico e de cálculo de vazão. Os valores propostos por Liddament (1986) são baseados na compilação de diversos estudos em túnel de vento e são recomendados para edifícios baixos (até 3 pavimentos), de planta quadrada e desobstruídos. São, contudo, constantemente utilizados para edifícios mais altos devido à falta de dados de referência de $\mathrm{C}_{\mathrm{p}}$.

No presente trabalho, o uso de CFD permitiu obter dados mais detalhados de $\mathrm{C}_{\mathrm{p}}$ do que os valores médios fornecidos pela literatura, bancos de dados e outros modelos preditivos de $\mathrm{C}_{\mathrm{p}}$. Um exemplo é fornecido na Figura 7, que mostra os resultados das simulações para janelas de 4 dormitórios (b14, a14, b11, a11), todas localizadas na mesma fachada e no pavimento térreo. Algumas conclusões obtidas pela análise dessa figura são descritas a seguir.

Conforme esperado, diferentes aberturas apresentam diferentes valores de $\mathrm{C}_{\mathrm{p}}$ para a mesma direção do vento. Por exemplo, quando o ângulo de ataque é igual a $0^{\circ}$, as janelas b14 e b11 possuem $\mathrm{C}_{\mathrm{p}}$ de aproximadamente 0,5 , valor aproximadamente 5 vezes superior ao das janelas a14 e a11.

A distribuição proposta por Liddament (1986) indica que o valor máximo de $\mathrm{C}_{\mathrm{p}}$ ocorre quando o ângulo de ataque é igual a $0^{\circ}$. Contudo, esse comportamento não é válido para pontos localizados longe do centro da fachada, como os pontos b14, a14, b11, a11. Nesses pontos, o $\mathrm{C}_{\mathrm{p}}$ máximo ocorre quando o ângulo de ataque apresenta um desvio de $+30^{\circ}$ ou $-30^{\circ}$ (ângulo de ataque $330^{\circ}$ na Figura 7) em relação à orientação da fachada. 


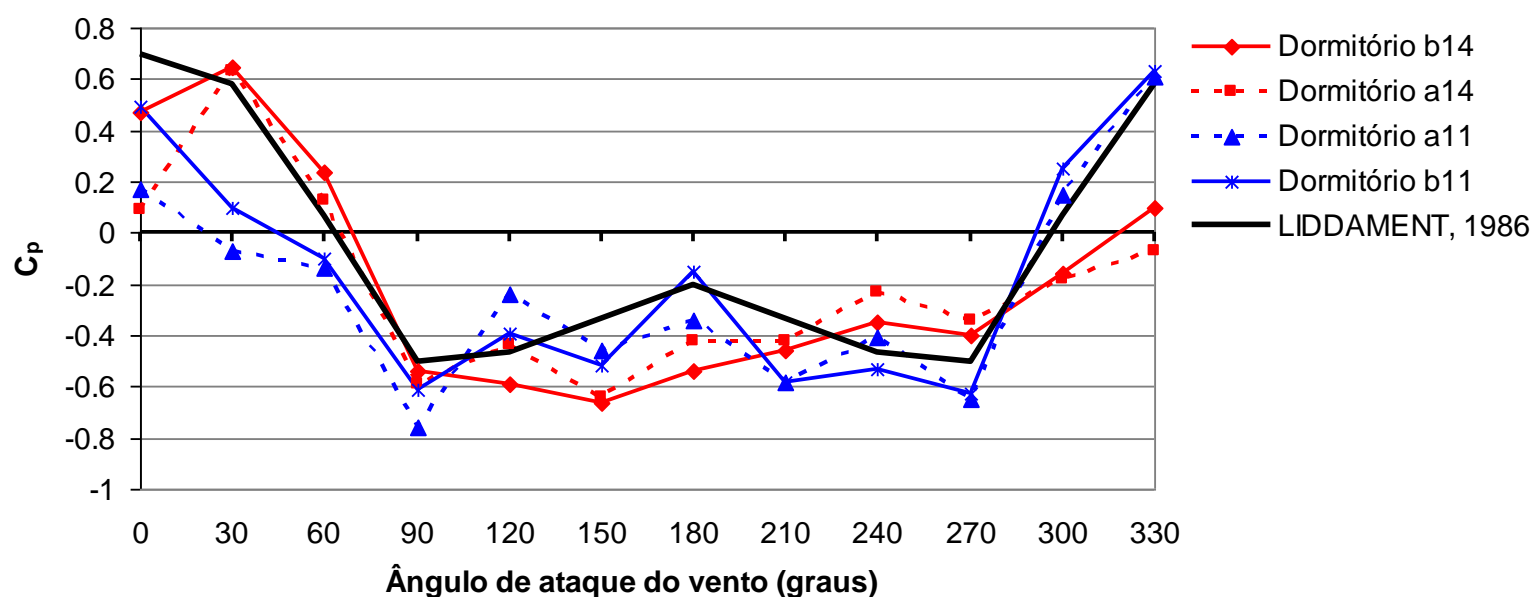

Figura 7 - Variação de $C_{p}$ em função do ângulo de ataque do vento, para pontos em uma mesma fachada e no mesmo andar
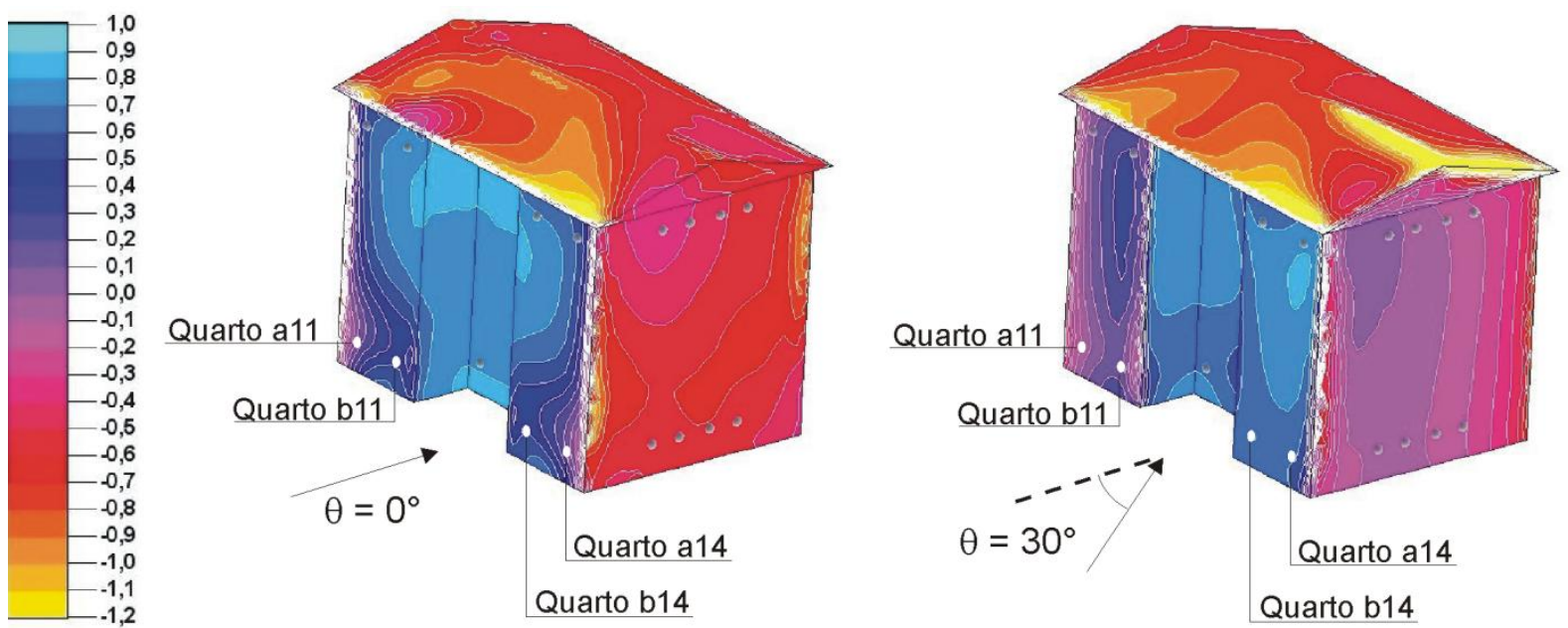

Figura 8 - Distribuição de $C_{p}$ para ângulos de ataque iguais a $0^{\circ}$ e a 30

Ainda de acordo com a Figura 7, quando o ângulo de ataque é igual a $60^{\circ}$, os pontos a14 e b14 apresentam pressão positiva, enquanto os pontos b11 e a11 apresentam pressão negativa. Esse comportamento não pode ser captado por valores de $C_{p}$ médio para a fachada, como os propostos por Liddament (1986).

A Figura 8 apresenta distribuições de $C_{p}$ para ângulos de ataque de $0^{\circ}$ e $30^{\circ}$ e assim possibilita a visualização de parte dos valores apresentados na Figura 7. Na Figura 8 é possível, por exemplo, verificar que, para o ângulo de ataque de $0^{\circ}$, as janelas b14 e b11 têm $\mathrm{C}_{\mathrm{p}} 5$ vezes maior que as janelas a14 e a11 por estarem mais próximas do centro da fachada. Com relação à ocorrência do $C_{p}$ máximo quando o ângulo de ataque é igual a $30^{\circ}$, a Figura 8 demonstra que o ponto de estagnação (ponto de maior pressão na fachada) é deslocado na direção dos pontos a14 e b14.

Com base nas Figuras 7 e 8 , é possível concluir que, quando comparados a valores médios obtidos em bancos de dados, valores de $\mathrm{C}_{\mathrm{p}}$ calculado usando CFD produzem informações valiosas sobre as diferenças de pressão entre pontos em uma mesma fachada.

\section{Conclusões}

Este trabalho procurou avaliar a viabilidade no uso de CFD para o cálculo de $\mathrm{Cp}$, assim como a importância na configuração de tais simulações.

A primeira conclusão aponta para a viabilidade no uso de CFD. A maior parte das simulações foi realizada sem maiores dificuldades em um computador pessoal. O tempo de processamento de $2 \mathrm{~h}$ para cada direção do vento permite que resultados para 12 direções do vento sejam obtidos em apenas 1 dia. Mesmo considerando o tempo de pré e pós-processamento, o custo e o tempo envolvidos em tais simulações são consideravelmente inferiores aos necessários à realização de experimentos em túnel de vento. 
Cabe salientar que esta pesquisa só pôde ser realizada porque diversas tarefas de pré e pósprocessamento foram automatizadas, e é essencial a adoção de procedimentos para aumentar a produtividade no uso do CFD.

A segunda conclusão deste trabalho remete à importância da correta configuração das simulações de CFD. Diferentes configurações levam a desvios consideráveis, tanto em termos absolutos (até $\pm 0,5$ ) quanto em termos relativos (até $50 \%$ ). O teste de independência da malha se mostrou fundamental para uma boa simulação, assim como a definição do perfil de vento. Nesse sentido, a divulgação ou a utilização de resultados de CFD sem os respectivos dados de entrada se mostram pouco recomendáveis, pois isso impede uma avaliação crítica quanto à qualidade e à confiabilidade dos resultados.

A revisão bibliográfica de práticas recomendadas em simulações de CFD juntamente com os resultados obtidos neste estudo possibilitaram a confecção de uma breve lista de aspectos que devem ser analisados em simulações de CFD. Essa lista foi elaborada considerando o uso de RANS, simulações em regime permanente, e constitui a principal contribuição deste trabalho para futuras pesquisas em CFD no contexto brasileiro:

(c) ordem de discretização das equações (preferencialmente de segunda ordem);

(d) teste de independência da malha (teste com pelo menos 3 malhas);

(e) $\mathrm{y}+$ (preferencialmente entre 30 e 100 para wall functions);

(f) modelo de turbulência (indicar o modelo utilizado);

(g) obstrução do domínio pelo objeto da simulação (preferencialmente igual ou inferior a $3 \%$ );

(h) dimensões do domínio em relação ao objeto da simulação (em caso de domínio circular, altura do domínio igual a 5 vezes a altura do edifício e distância de pelo menos 10 vezes a altura do edifício nas demais direções);

(i) condição de contorno das fronteiras sólidas (descrever a condição de escorregamento free slip ou no slip, especificar o uso de wall functions ou low Reynolds number e, quando aplicável, informar a rugosidade adotada);

(j) condições de contorno nas fronteiras fluidas (especificar o tipo de abertura pressure, velocity ou opening, e, quando aplicável, descrever o perfil de velocidade incluindo a rugosidade ou expoente utilizados e a intensidade de turbulência); e (k) resíduo (necessariamente igual ou inferior a $\left.10^{-4}\right)$.

Com relação aos modelos de turbulência baseados em RANS e disponíveis para simulações de CFD, é notável a necessidade de mais estudos para clarificar as reais vantagens no uso de modelos de fechamento de segunda ordem. São também necessários mais estudos sobre o desempenho de diferentes modelos de fechamento de primeira ordem com relação ao cálculo de $\mathrm{C}_{\mathrm{p}}$, especialmente em face do declínio do uso do modelo standard $\mathrm{k}-\varepsilon$ e do uso mais frequente dos modelos realizable $\mathrm{k}-\varepsilon$, RNG $\mathrm{k}-\varepsilon$ ou SST k- $\omega$ em aplicações de engenharia de vento.

O uso de práticas descritas na literatura apenas garante que a simulação de CFD foi realizada adequadamente, contudo seus resultados devem ser necessariamente validados antes de qualquer utilização para fins práticos. Portanto, futuros trabalhos devem se concentrar na validação das simulações através da comparação sistemática com ensaios em túnel de vento previamente realizados, tais como os reportados por Quan et al. (2007), e pela comparação com medições em edifícios reais.

Cabe salientar que, em diversas situações, o edifício cuja ventilação natural se pretende analisar pode não ser sensível às variações no valor de $C_{p}$. Edifícios em áreas com baixas velocidades do vento, por exemplo, são pouco afetados por variações em $\mathrm{C}_{\mathrm{p}}$. Nesses casos, é dispensável despender maiores esforços na obtenção de valores de $C_{p}$ mais precisos.

\section{Referêncais bibliográficas}

\author{
AIR INFILTRATION AND VENTILATION \\ CENTER (AIVC). In: WIND PRESSURE \\ WORKSHOP AIC, Brussels, 1984. Proceedings... \\ Brussels: AIVC, 1984.
}

ALLARD, F. (Ed.). Natural Ventilation in

Buildings: a design handbook. London: James X James, 1998.

AMERICAN SOCIETY OF HEATING,
REFRIGERATING AND AIR-CONDITIONING
ENGINEERS (ASHRAE). ASHRAE Handbook:
fundamentals. Atlanta: ASHRAE, 2001 .

AWBI, H. B. Ventilation of Buildings. London: E \& FN Spon, 1998a.

AWBI, H. B. Calculation of Convective Heat Transfer Coefficients of Room Surfaces for Natural Convection. Energy and Buildings, v. 28, n. 2, p. 219-227, 1998 b. 
BITTENCOURT, L.; CÂNDIDO, C. Introdução à Ventilação Natural. Maceió: Edufal, 2008.

BLOCKEN, B.; CARMELIET, J. The Influence of the Wind-Blocking Effect by a Building on its Wind-Driven Rain Exposure. Journal of Wind Engineering and Industrial Aerodynamics, v. 94, n. 2, p. 101-127, 2006.

BLOCKEN, B.; STATHOPOULOS, T.; CARMELIET, J. CFD Simulation of the Atmospheric Boundary Layer: wall function problems. Atmospheric Environment, v. 41, n. 2, p. 238-252, 2007.

BRANDÃO, R. S. As interações Espaciais Urbanas e o Clima. 2009. Tese (Doutorado em Arquitetura e Urbanismo) - Faculdade de Arquitetura e Urbanismo, Universidade de São Paulo, São Paulo, 2009.

CÂNDIDO, C.; TORRES, S.; BITTENCOURT, L. Avaliação do Uso de Peitoril Ventilado para Melhorar a Ventilação Natural em Edificações: estudo de caso em núcleo de pesquisa multidisciplinar em Maceió, AL. In: ENCONTRO NACIONAL DE CONFORTO DO AMBIENTE CONSTRUÍDO, 7., Maceió, 2005. Anais... Maceió: ANTAC, 2005. 1 CD-ROM.

CARDOSO, S.; PESSOA, I.; BITTENCOURT, L. Avaliação de Utilização de Captadores de Vento Numa Edificação da Universidade Federal de Alagoas. In: ENCONTRO NACIONAL DE CONFORTO DO AMBIENTE CONSTRUIIDO, 7., Maceió, 2005. Anais... Maceió: ANTAC, 2005. 1 CD-ROM.

CAVALCANTI, F.; CÂNDIDO, C.; BITTENCOURT, L. Avaliação da Ventilação Natural em Espaços de Sala de Aula a Partir da Implantação de Pérgulas. In: ENCONTRO NACIONAL DE CONFORTO DO AMBIENTE CONSTRUÍDO, 7., Maceió, 2005. Anais... Maceió: ANTAC, 2005. 1 CD-ROM.

CFX. CFX 5 Solver Models. Diddcot Oxfordshire: CFX, 2003.

COST. Cost Action 14: recommendations on the use of CFD in predicting pedestrian wind environment. Brussels: COST, 2004.

CÓSTOLA, D. Ventilação por Ação do Vento no Edifício: procedimentos para quantificação. 2006. 214 f. Dissertação (Mestrado em Arquitetura e Urbanismo) - Faculdade de Arquitetura e Urbanismo, Universidade de São Paulo, São Paulo, 2006.
CÓSTOLA, D.; BLOCKEN, B.; HENSEN, J. Overview of Pressure Coefficient Data in Building Energy Simulation and Airflow Network Programs. Building and Environment, v. 44, n. 10, p. 2027-2036, 2009.

CÓSTOLA, D. et al. Uncertainty in Airflow Rate Calculations Due to the Use of Surface-Averaged Pressure Coefficients. Energy and Buildings, v. 42, n. 6, p. 881-888, jun. 2010.

CRAWLEY, D. et al. Contrasting the Capabilities of Building Energy Performance Simulation Programs. Building and Environment, v. 43, n. 4, p. 661-673, 2008.

DAVIDSON, P. A. Turbulence: an introduction for scientists and engineers. Oxford: Oxford University Press, 2004.

DEFRAEYE ,T.; BLOCKEN, B.; CARMELIET, J. CFD Analysis of Convective Heat Transfer at the Surfaces of a Cube Immersed in a Turbulent Boundary Layer. International Journal of Heat and Mass Transfer, v. 53, n. 1/3, p. 297-308, 2010.

ETHERIDGE, D. W.; SANDBERG, M. Building Ventilation: theory \& measurement. Chichester: John Wiles \& Sons, 1996.

FIGUEIREDO, C. M. Ventilação Natural para Conforto Térmico em Edifícios de Escritórios. 2008. Dissertação (Mestrado em Arquitetura e Urbanismo) - Faculdade de Arquitetura e Urbanismo, Universidade de São Paulo, São Paulo, 2008.

FRANKE, J. et al. Cost Action 732: best practice guideline for the CFD simulation of flows in the urban environment. Brussels: COST, 2007.

FROTA, A. B.; SCHIFFER, S. R. Manual de Conforto Térmico: arquitetura e urbanismo. São Paulo: Studio Nobel, 2000.

GROSSO, M. Wind Pressure Distribution Around Buildings: a parametrical mode. Energy and Buildings, v. 18, p. 101-131, 1992.

HARRIES, A. Notas de Aula. In: WORKSHOP: CFX - FAU-USP. São Paulo:[s.n.], 2005.

HEIJMANS, N.; WOUTERS, P. IEA Impact of the uncertainties on wind pressures on the prediction of thermal comfort performances: technical report. HybVent Annex35, Brussels, [1995?].

HOOFF, T. van; BLOCKEN, B. Coupled Urban Wind Flow and Indoor Natural Ventilation Modelling on a High-Resolution Grid: a case study for the Amsterdam ArenA stadium.

Environmental Modelling \& Software, v. 25, n. 1, p. 51-65, 2010. 
HOLSCHER, N.; NIEMANN, H. J. Towards Quality Assurance for Wind Tunnel Tests: a comparative testing program of the Windtechnologische Gesellschaft. Journal of Wind Engineering and Industrial Aerodynamics, v. 74, p. 599-608, 1998.

IRMINGER, J. O. V.; NØKKENTVED, C. H. R. Wind-Pressure on Buildings. Copenhagen: Danmarks Naturvidenskabelige Samfund, 1936.

JENSEN, M.; FRANCK, N. Model-Scale Tests in Turbulent Wind:part II. Copenhagen: Danish Technical Press, 1965.

KNOLL, B.; PHAFF, J. C.; GIDS, W. F. Pressure Simulation Program. In: CONFERENCE ON IMPLEMENTING THE RESULTS OF VENTILATION RESEARCH, 16., Palm Spring, 1995. Proceedings... Palm Spring: AIVC, 1995.

LEITE, C. G. Alterações da Ventilação Urbana Frente ao Processo de Verticalização de Avenidas Litorânea. 2008. Dissertação (Mestrado em Arquitetura e Urbanismo) - Faculdade de Arquitetura e Urbanismo, Universidade de São Paulo, São Paulo, 2008.

LIDDAMENT, M. W. Air Infiltration Calculation Techniques: an applications guide. Bracknell: AIVC, 1986.

MONTEIRO, L. M. et al. Conforto Térmico como Condicionante do Projeto ArquitetônicoPaisagístico: o caso dos espaços abertos do novo centro de pesquisas da Petrobras no Rio de Janeiro, CENPES II. Ambiente Construído, Porto Alegre, v. 8, n. 4, p. 61-86, out./dez. 2008.

NOZU, T. et al. LES of the Flow and Building Wall Pressures in the Center of Tokyo. Journal of Wind Engineering and Industrial Aerodynamics, v. 96, n. 1, p. 1762-1773, 2008.

ORME, M. L. Applicable models for air infiltration and ventilation calculations: technical note 51. [s.1.]: AIVC, 1999.

PRATA, A. R. Impacto da Altura de Edifícios nas Condições de Ventilação Natural do Meio Urbano. 2005. $271 \mathrm{f}$. Tese (Doutorado em Arquitetura e Urbanismo) - Faculdade de Arquitetura e Urbanismo, Universidade de São Paulo, São Paulo, 2005.
QUAN, Y. et al. TPU Aerodynamic Database for Low-Rise Buildings. In: INTERNATIONAL CONFERENCE ON WIND ENGINEERING, 12 ., Cairns, 2007. Proceedings... Cairns, 2007.

REINHOLD, T. A. Wind Tunnel Modelling for Civil Engineering Applications. Cambridge: Cambridge University Press, 1982.

RICHARDS, P. J.; HOXEY, R. P.; SHORT, L. J. Wind Pressures on a $6 \mathrm{~m}$ Cube. Journal of Wind Engineering and Industrial Aerodynamics, v. 89, p. 1553-1564, 2001.

SANTAMOURIS, M.; WOUTERS, P. Building Ventilation: the state of the art. London: Earthscan, 2006.

SIMPSON, C.; WHALLEY, M. Dynamic Thermal Modelling and Physical Wind Tunnel Testing: an important partnership for building simulation. In: BUILDING SIMULATION, 2009, Glasgow, Scotland. Proceedings... Glasgow, Scotland, 2009.

STATHOPOULOS, T. Computational Wind Engineering: past achievements and future challenges. Journal of Wind Engineering and Industrial Aerodynamics, v. 67/68, n. 509-532, p. 509-532, 1997.

STULL, R. An Introduction to Boundary Layer Meteorology. Dordrecht: Kluwer Academic Publishers, 1998.

SWAMI, M. V.; CHANDRA, S. Correlations for Pressure Distribution on Buildings and Calculation of Natural-Ventilation Airflow. ASHRAE

Transactions, v. 94, n. 1, p. 243-266, 1988.

TOLEDO, E. Ventilação Natural de Edifícios. Lisboa: LNEC, 1967.

WIT, S. Uncertainty in Predictions of Thermal Comfort in Buildings. 2001. Thesis (PhD Thesis) - Delft University of Technology, 2001.

YANG, T. CFD and Field Testing of a Naturally Ventilated Full-Scale Building. Thesis (PhD Thesis) - School of Civil Engineering, University of Nottingham, 2004.

\section{Agradecimentos}

Os autores agradecem ao $\mathrm{CNPq}$, pelo financiamento desta pesquisa. Agradecem também a Alan Harris, pelo suporte com o CFX, e a Bert Blocken, Wiebe Zoon e Mohammad Mirsadeghi, pelas discussões sobre CFD. 
Revista Ambiente Construído

Associação Nacional de Tecnologia do Ambiente Construído Av. Osvaldo Aranha, $99-3^{\circ}$ andar, Centro

Porto Alegre - RS - Brasil

CEP $90035-190$

Telefone: +55 (51) 3308-4084

Fax: +55 (51) 3308-4054

www.seer.ufrgs.br/ambienteconstruido

E-mail: ambienteconstruido@ufrgs.br 